\title{
Density- and serum-dependent regulation of the Reck tumor suppressor in mouse embryo fibroblasts.
}

\section{$\operatorname{AUTHOR}(\mathrm{S})$ :}

Hatta, Mamiko; Matsuzaki, Tomoko; Morioka, Yoko; Yoshida, Yoko; Noda, Makoto

\section{CITATION:}

Hatta, Mamiko ...[et al]. Density- and serum-dependent regulation of the Reck tumor suppressor in mouse embryo fibroblasts.. Cellular signalling 2009, 21(12): 1885-1893

\section{ISSUE DATE:}

2009-12

URL:

http://hdl.handle.net/2433/128757

\section{RIGHT:}

C 2009 Elsevier B.V.; この論文は出版社版でありません。引用の際には 出版社版をご確認ご利用ください。; This is not the published version. Please cite only the published version. 


\section{Density-dependent up-regulation of Reck tumor suppressor protein in mouse embryo fibroblasts}

Mamiko Hatta, Tomoko Matsuzaki, Yoko Morioka, Yoko Yoshida and Makoto Noda*

Department of Molecular Oncology, Kyoto University Graduate School of Medicine, Yoshida-Konoe-cho, Sakyo-ku, Kyoto 606-8501, Japan

Keywords:

Mmp2, fibroblasts, Src, PI3K, collagen, fibronectin

* Corresponding author. Department of Molecular Oncology, Kyoto University Graduate School of Medicine, Yoshida-Konoe-cho, Sakyo-ku, Kyoto 606-8501, Japan. Tel.: +81-75-751-4150; fax. +81-75-751-4159.

E-mail address: mnoda@z06.mbox.media.kyoto-u.ac.jp (M. Noda) 


\section{ABSTRACT}

Reck is a membrane-anchored glycoprotein initially identified as a transformation suppressor. Accumulating evidence indicates that Reck negatively regulates a wide spectrum of matrix metalloproteinases and is commonly down-regulated in malignant solid tumors. Physiological cues that regulate RECK expression, however, remained unknown. In this study, we found that RECK expression in mouse embryo fibroblasts increased at high cell density, after serum-deprivation, and in the cells treated with two kinase inhibitors, PP2 (Src inhibitor) and LY294002 (PI3-kinase inhibitor). Curve fitting indicated that the level of Reck protein as well as its mRNA could be approximated by a quadratic function of cell density. Other stimuli, including serum-deprivation, extracellular matrix components (type I collagen and fibronectin), the kinase inhibitors, and their oncogenic targets ( $v$-Src and PIK3CA E545K), modify the shape of this quadratic curve. Comparison of these modifications suggest that Src is involved in the Reck down-regulation under sparse conditions while PI3-kinase is involved in the serum-induced Reck down-regulation. Fibronectin and type I collagen may counteract with the sparsity-Src signaling and the serum-PI3-kinase signaling, respectively. These findings provide a framework for further studies to clarity the molecular mechanisms of Reck gene regulation and to find effective Reck inducers of potential value in cancer therapy. 


\section{Introduction}

Mammalian cells rely on both genetic program and environmental cues in deciding their fates and behaviors. In mouse embryo fibroblasts (MEFs), for instance, lifespan is pre-determined by genetic program (senescence), and yet, even in their early passages, the cells stop dividing when they lack enough growth factors, anchorage, or space. Cancer cells are often less sensitive to these environmental restrictions.

Important external stimuli influencing cell fate-decision include humoral factors, extracellular matrix (ECM), and adjacent cells. A large body of knowledge has been accumulated on the mechanisms of signal transduction through membrane-associated receptors for various humoral factors and ECM components. For instance, receptor tyrosine kinases transmit signals of various growth factors by activating multiple signaling cascades [1, 2], including Ras/Erk and phosphatidyl inositol 3-kinase (PI3K)-Akt pathways [3, 4]. Integrins, a major family of ECM receptors, transmit signals through two non-receptor type tyrosine kinases, Fak and Src, to activate several down-stream signaling pathways, including Cas/Jnk, Ras/Erk, and Rho/Rac/Cdc42 pathways [5]. Hence, we now begin to understand how the growth factor cues and the ECM cues are integrated to provoke proper cell responses in normal cells, and how these mechanisms are deficient in cancer cells. In contrast, the mechanisms by which the cells sense space and avoid one another are still unclear, despite their obvious importance in understanding various biological events, such as animal development, wound-healing, and carcinogenesis.

As an approach to understand how oncogenes induce transformation, a breakdown of the contact-inhibited growth regulation, we previously screened genes inducing flat reversion (i.e., restored contact-inhibition) in $v$-K-ras-transformed NIH3T3 cells [6]. One such gene, RECK, was found to encode a unique membrane-anchored inhibitor of matrix metalloproteinases (MMPs) [7]. The level of RECK expression is low in fibroblasts after transformation by various oncogenes and in a number of cell lines derived from various cancer tissues [8]. Moreover, forced expression of RECK in cancer cells resulted in suppression of tumor angiogenesis, invasion, and metastasis in animal xenograft models [9]. Subsequent studies with clinical samples not only confirmed frequent down-regulation of RECK in a wide variety of solid tumors but also revealed significant correlation between the level of residual RECK expression in tumors and better prognosis [10].

The strong conservation of Reck from fruit fly to man as a single gene seems to suggest its pivotal physiological functions. In fact, Reck-deficient mice die around embryonic day 10.5 with reduced tissue integrity and defects in various tissues 
including blood vessels and the neural tubes [9, 11], suggesting its essential role in animal development. Possible involvements of Reck in the development of skeletal muscles, cartilage, and the neuromuscular junctions have also been suggested [12-14]. At the single cell level, RECK is required for stable adhesion, persistent migration, and endocytic inactivation of membrane-bound proteases [15]. Hence, knowledge on the physiological stimuli that regulate RECK expression are of great importance in understanding a wide range of biological events including cancer progression, mammalian development, and cell migration. Such knowledge may also be useful in developing the methods to re-activate RECK expression in cancer cells and suppress malignancy.

In the present study, we examined the effects of cell density, serum, ECM, several inhibitors, and some of their targets on Reck expression in mouse embryo fibroblasts. Quantitative analysis of these data revealed potential relationships among these external stimuli and signaling molecules in the density-dependent regulation of Reck expression.

\section{Materials and methods}

\subsection{Cell culture}

NIH3T3 and v-Src NIH (2-1/NP; a gift from Steve Anderson, University of Colorado Health Science Center) were maintained in Dulbecco's modified Eagle medium (DMEM) supplemented with $10 \%$ fetal bovine serum and antibiotics (referred to as "growth medium" in this paper). MEF and M2 MEF were established as described elsewhere [16] and maintained in aMEM supplemented with 10\% FBS and antibiotics. NIH3T3 cells were transfected with mammalian expression vector pcDNA6-PIK3CA E545K (a gifts from Masahiro Aoki, Kyoto university Graduate School of Medicine) and selected in medium containing $8 \mu \mathrm{g} / \mathrm{ml}$ blastcitidine-S to establish PIKm NIH. To determine growth curves, NIH3T3 or MEF cells were plated onto thirty $35 \mathrm{~mm}$-dishes at $5 \times 10^{4}$ cells per dish. Two dishes were used for cell counting, one dish for protein analysis, and the other dishes were refed with fresh medium every day. To arrest cell cycle, NIH3T3 cells were plated at $1 \times 10^{5}$ cells per 60 -mm dish with growth medium. After overnight incubation, the cells were exposed to serum-free DMEM for $24 \mathrm{~h}$ or to the growth medium containing hydroxyurea $(10 \mathrm{mM})$ or nocodazole $(100 \mathrm{ng} / \mathrm{ml})$ for $16 \mathrm{~h}$. For double thymidine-block, the cells plated on the previous day were incubated for $16 \mathrm{~h}$ in DMEM containing $2 \mathrm{mM}$ thymidine, for $8 \mathrm{~h}$ in growth medium, followed by the second $16 \mathrm{~h}$ incubation in DMEM containing $2 \mathrm{mM}$ thymidine. Dishes coated with type I collagen were obtained from IWAKI, and plasma fibronectin was from Sigma. 


\subsection{Inhibitors}

PP2, LY294002, PD98059, GF109203x, Y27632, and Toxin B were obtained from Calbiochem; hydroxyurea, nocodazole, thymidine, 5-azacitidine, and Trichostatin A were from Sigma.

\subsection{Immunoblot assay}

To extract proteins, culture cells were lysed in 50mM Tris- $\mathrm{HCl}$ (pH6.8), 1\% SDS, $1 \mathrm{mM}$ EDTA, and the protease inhibitor cocktail (Nakarai Tesque). Proeins were separated by SDS-PAGE ( $8 \%$ acrylamide), followed by immunoblot detection using monoclonal antibody against RECK (5B11D12) and re-probing with anti- $\alpha$-tubulin (Calbiochem) or anti-Gapdh (Ambion). Immobilon Western (Millipore) was used for visualization. Densitometry was performed using ImageJ64 software.

\subsection{Quantitative real-time PCR}

Total RNA was extracted using Nucleo Spin RNA II (MACHEREY-NAGEL). The amount of mRNAs was measured using SuperScript III Platinum One-Step Quantitative RT-PCR System (Invitrogen) or SuperScript III Platinum SYBR Green One-Step qRT-PCR Kit (Invitrogen) on Mx 3005P Real-Time PCR System /Mx Pro software (Stratagene). Primer pairs and probes for mouse Reck: 5'-AGGTCTCCAGCAGTCTCC-3', 5'-GCAGTTCCTTCCAGTTGTG-3', and 5'(FAM)-CGCGATCCCACTCCCTGCTCCTCCTCCTCAGATCGCG-(Eclipse)3'

(Molecular beacon probe). Primer pairs for the control mouse hypoxanthine phosphoribosyl-transferase 1 (Hprt 1): 5'-AGCGTTTCTGAGCCATTGC-3' and 5'-GCGGTCTGAGGAGGAAGC-3'. Reaction conditions: initial reactions at $50^{\circ} \mathrm{C}$ for $5 \mathrm{~min}$ and at $95^{\circ} \mathrm{C}$ for $5 \mathrm{~min}$ followed by 45 cycles of reactions at $94^{\circ} \mathrm{C}$ for $5 \mathrm{~min}$, at $55^{\circ} \mathrm{C}$ for $40 \mathrm{~s}$, and at $72^{\circ} \mathrm{C}$ for $15 \mathrm{~s}$.

\subsection{Data analyses}

The values obtained by densitometry (protein) or qRT-PCR (mRNA) were first divided by the values of internal standard (normalized data) and then divided by the normalized data for NIH3T3 cells plated at $5 \times 10^{5}$ cells per $35-\mathrm{mm}$ dish and incubated in medium containing 10\% FBS (termed "reference sample"). The obtained value was termed "relative Reck level" or $(\boldsymbol{r})$. The $\boldsymbol{r}$ values were plotted against cell density ( $\boldsymbol{d}$ [x10 ${ }^{6}$ cells per $35-\mathrm{mm}$ dish]) using Excel 2008 software, and approximation curves with highest $\mathrm{R}^{2}$ values were searched among the standard options. Second order 
polynomial approximation was the best choice in all cases tested. The coefficients (p, q) of the standard form $\left[\mathrm{y}=\mathrm{a}(\mathrm{x}-\mathrm{p})^{2}+\mathrm{q}\right]$ of the $\boldsymbol{r}-\boldsymbol{d}$ approximation equation $(\mathrm{y}=\mathrm{ax}+\mathrm{bx}+\mathrm{c})$ were calculated using the following formula: $p=-b / 2 a, q=\left(4 a c-b^{2}\right) / 4 a$.

\section{Results}

\subsection{Reck is up-regulated at high cell density in fibroblasts}

In our previous studies using various cells in culture, we noticed that the expression of endogenous RECK can fluctuate to a large extent. For instance, when a commonly used mouse embryo fibroblast line, NIH3T3, was cultured for 10 days starting from sparse conditions, the level of Reck protein increased dramatically as time passed and the cells proliferated (Fig. 1A, black diamond, and Fig. 1B). Similar up-regulation of Reck protein was found in a newly established mouse embryo fibroblast line (MEF) derived from a wild type mouse embryo (Fig. 1A, white diamond, and Fig. 1C). MEF resembles primary fibroblasts in its low clonogenicity, very low transfection efficiency, and higher persistence during long term culture. To clarify whether the Reck up-regulation depends on cell density or some other factors associated with prolonged incubation, we plated NIH3T3 or MEF at several different cell densities and incubated for $48 \mathrm{~h}$. The data clearly demonstrated density-dependent up-regulation of Reck both in NIH3T3 (Fig. 1D, black bars) and MEF (Fig. 1D, white bar). We also examined the regulation of Reck expression in another fibroblast line, M2 MEF, derived from a mouse embryo lacking one of the Reck targets, Mmp-2 (Fig. 1D, striped bars). Although this cell line also show density-dependent Reck regulation, the level of Reck was significantly higher as compared to that in MEF or NIH3T3. Measurement of Reck mRNA by quantitative reverse transcription-polymerase chain reaction (q-RT-PCR) revealed that the density-dependent up-regulation of Reck in these cell lines was seen at the mRNA level (Fig. 1E), although the proportion of Reck protein (Fig. 1D) and Reck mRNA (Fig. 1E) seems different among cell lines, suggesting different degree of post-transcriptional regulation among the cell lines.

\subsection{Reck is up-regulated after serum-deprivation in fibroblasts}

We noticed that the concentration of fetal bovine serum ("serum") in culture medium also affected Reck expression. When NIH3T3 cells plated at the same cell density were incubated in medium containing various concentrations of serum for $24 \mathrm{~h}$, clear inverse correlation between serum concentration and Reck protein level was observed (Fig. 2A). This regulation seems to also operate at the mRNA level (Fig. 2B). Time course studies suggested that this up-regulation took place rather slowly: Reck 
level continued to build up over $24 \mathrm{~h}$ (Fig. 2C). Decline of Reck level after $24 \mathrm{~h}$ serum-deprivation also continued at least for $24 \mathrm{~h}$ (Fig. 2D).

\subsection{Effects of various inhibitors on Reck expression}

Since high cell density and serum-deprivation both lead to cell cycle arrest, we suspected that Reck expression might be commonly activated when cell cycle was arrested. To test this possibility, we monitored the level of Reck protein in NIH3T3 cells after double thymidine-block (Td; cell cycle arrest at G1/S boundary), treatment with hydroxyurea (HU; cell cycle arrest in S phase), or with nocodazole (Nz; cell cycle arrest in $\mathrm{G} 2 / \mathrm{M})$. These treatments, however, showed little effects on Reck expression (Fig. 3A), supporting the idea that specific environmental cues (e.g., high cell density and serum-deprivation), rather than cell cycle-arrest, are responsible for Reck up-regulation.

Previous studies indicated that RECK expression in certain transformed or cancer-derived cells could be induced by treatment with a DNA methyltransferase inhibitor, 5-azacytidine $(\mathrm{AzC})$, or histone deacetylase inhibitor, trichostatin A (TSA) [17-20] . To test whether DNA methylation and/or histone acetylation play any roles in the density- and/or serum-dependent regulation of Reck expression in NIH3T3 cells, we treated the cells at low cell density $\left(2 \times 10^{4}\right.$ per $35 \mathrm{~mm}$-dish) with these inhibitors in the presence of $10 \%$ serum (Fig. 3B). Reck expression was induced by TSA but not by AzC (Fig. 3B, lanes 1-3), suggesting that histone deacetylation, but not DNA methylation, may be involved in the mechanism to keep Reck expression relatively low under these conditions (i.e., low density, high serum). Moreover, the effects of TSA were marginal at high cell density (Fig. 3C, lane 4) and can even be suppressive in serum-free medium (Fig. 3C, lanes 6, 8), supporting the idea that under such conditions, histones are abundantly acetylated, so that the histone deacylase inhibitor (TSA) cannot exert strong, positive effects on Reck expression.

To find clues to the signaling pathways affecting Reck expression, we also tested the effects of some selective inhibitors targeting molecules involved in the integrin or growth factor signaling. The inhibitors we used (and their targets) are as follows: GF109203x (protein kinase C $\alpha, \beta I, \beta I I, \gamma, \delta, \varepsilon$ ), PP2 (Src), PD98059 (Mek1), LY294002 (PI3K), Y27632 (Rock), and ToxinB (Rac, Cdc42). When NIH3T3 cells at low cell density were treated with sub-toxic doses of each drug for $48 \mathrm{~h}$ in the presence of $10 \%$ serum, significant changes in Reck expression could be detected after treatment with GF109203x, PP2, or LY294002 (Fig. 3D). PP2 and LY294002 co-operatively up-regulated Reck at low cell density in the presence of $10 \%$ serum (Fig. 3E). 


\subsection{Relationship between cell density and serum-deprivation in the regulation of Reck expression}

Having found the strong influence of cell density and serum concentration on Reck expression, we attempted to clarify the extent of, and the relationship between, the effects of these two factors on Reck. NIH3T3 cells were plated at a series of different cell densities, incubated in medium containing $10 \%$ serum or in serum-free medium for $24 \mathrm{~h}$, and the level of Reck protein was estimated by immunoblot assay (Fig. 4A) followed by densitometry. In this and following experiments, the "relative level of Reck (r)" was defined as the level of Reck protein or mRNA normalized against a loading control, $\alpha$-tubulin or Hprt mRNA, and then divided by the data for a reference sample [i.e., NIH3T3 cells at plating density $(\boldsymbol{d})$ of $5 \times 10^{5}$ per $35 \mathrm{~mm}$-dish in $10 \%$ serum]. When we plotted $\boldsymbol{r}$ against $\boldsymbol{d}$, the data for the cells at both serum concentrations were found to fit 2 nd order polynomial approximation curves (i.e., parabola) with relatively high accuracy $\left(\mathrm{R}^{2}>0.97\right)$ (Fig. 4B). This means that $\boldsymbol{r}$ can be approximated by a quadratic function of $\boldsymbol{d}$. The differences in shape and position between the two quadratic curves suggest that serum affects multiple coefficients of this function.

To assess the feasibility of using such curve-fitting technique in evaluating the effects of various treatments on Reck expression, we first analyzed the data from five independent control experiments [i.e., measurements of Reck in NIH3T3 cells at a series of cell densities in the presence of $10 \%$ serum]. The profile of 5 coefficients ("a, b, c, p, q" described Fig. 4C) was found to stay reasonably constant (Fig. 4D, black bars), supporting the idea that any significant change in the profile after certain treatment may be considered meaningful. The averaged "Reck protein-cell density" (or $\boldsymbol{d}_{\boldsymbol{p}}-\boldsymbol{r}$ ) relationship for NIH3T3 was as follows: $\boldsymbol{r}=-0.547 \boldsymbol{d}^{2}+1.874 \boldsymbol{d}+0.1296$ [or $\boldsymbol{r}=$ $\left.1.735-0.547(\boldsymbol{d}-1.713)^{2}\right]$.

\subsection{Reck regulation in MEFs and after treatment with kinase inhibitors}

Similar analyses of the data shown in Fig. 1D indicated that the $\boldsymbol{d}_{\boldsymbol{p}}-\boldsymbol{r}$ relationship for MEF and M2 MEF also fit quadratic approximation curves, albeit with altered shapes (Fig. 4E). Hence, the quadratic model may be applicable to multiple fibroblastic cell lines. Moreover, both of the curves show vertexes, an important feature of parabola, providing additional evidence supporting quadratic model.

The protein kinase inhibitors, PP2 and LY294002, seem to have effects distinct from each other on the $\boldsymbol{d}_{\boldsymbol{p}} \boldsymbol{- r}$ curve. The PP2-treated cells gave rise to a flatter curve (Fig. 5A), while the LY294002-treated cells causes left-ward shift of the curves (Fig. 5B) as 
compared to the control data. Hence, although these chemicals seemed to have similar effects on Reck expression when tested at a relatively low cell density (Fig. 3D, E), their modes of action probably differ from each other, as we noticed only when their effects were tested at a series of different cell densities.

\subsection{Effects of ECM components and oncogenes on Reck mRNA expression}

As we compare the cells on different substrates (e.g., dishes coated with ECM components), the contaminating substrate components may hinder accurate protein determination required for quantitative immunoblot assay. In transformed cells, elevated proteolytic activity makes it difficult to measure the level of Reck protein accurately by immunoblot-densitometry. In such cases, estimation of Reck expression at the mRNA level may be more reliable. Comparison among three independent control experiments [i.e., measurements of Reck mRNA by qRT-PCR in NIH3T3 plated at a series of different densities in the presence of $10 \%$ serum] revealed that the profile of coefficients were similar to that for Reck protein and reasonably constant (Fig. 4D, compare white bars with black bars). Thus, comparison of these coefficients at the mRNA level may also be useful in assessing the effect of various treatments on Reck expression. The averaged "Reck mRNA-cell density" (or $\boldsymbol{d}_{\boldsymbol{r}} \boldsymbol{- r}$ ) relationship in NIH3T3 was as follows: $\boldsymbol{r}$ $=-0.7675 \boldsymbol{d}^{2}+1.817 \boldsymbol{d}+0.1761$ [or $\left.\boldsymbol{r}=1.251-0.7675(\boldsymbol{d}-1.184)^{2}\right]$.

Using this approach, we found an interesting distinction between the effects of type I collagen (Coll-1; Fig. 5C) and fibronectin (FN; Fig. 5D) on Reck mRNA expression. Coll-1 yielded more closed parabola, whereas FN caused downward shift of the curve. Hence, in the lower cell density range, Coll-1 and FN have opposing effects.

We also examined the effects of v-Src [a constitutively active form of PP2 target] and PIK3CA E545K (PIKm) [an oncogenic form of a subunit of LY294002 target] to see whether these genes show effects opposite to PP2 and LY294002, respectively. The effects of v-Src were striking: almost complete loss of density-dependent Reck up-regulation (Fig. 5E). The effects of PIKm were milder: more open parabola with slightly lower vertex and rightward shift (Fig. 5F).

\section{Discussion}

What can we learn from these data? For quantitative comparison, four coefficients (a, c, p, q) of the $\boldsymbol{d}$-r curves at the protein level (Fig. 6A) and the mRNA level (Fig. 6B) are summarized. We chose these coefficients, since they directly determine the basic features of parabola: a) its shape or closeness, c) y-intercept, p) axis of symmetry, and q) height of vertex. We adopted $-\mathbf{a}$ (or lal) to make the values positive and to make it 
possible to compare all coefficients in one graph. To facilitate comparison among the coefficients, the values relative to those for the control group [i.e., NIH3T3 in 10\% serum] are presented. The trends found in these graphs are summarized in Fig. 6C.

At first sight, it seems evident that the value $\mathbf{c}$ is relatively high in most of the cases where we found Reck up-regulation. This, however, is likely to be a consequence of, rather than a clue to, the mechanism by which Reck expression is modulated, since we focus on the treatments effective in up-regulating Reck expression in a low-cell-density range (i.e., $\mathbf{d}$ close to zero) in this study.

Upon close inspection of these coefficients (Fig. 6A-C) and the d-r curves (Fig. 4 5), however, we noticed that there were at least three ways to up-regulate Reck expression at low cell density (Fig. 6D): (1) to make parabola more open by lowering lal [PP2], (2) to close parabola by increasing lal plus lowering p $[0 \%$ serum, MEF, M2 MEF, LY294002, and Coll-1], and (3) to shift the curve upward by increasing $\mathbf{q}[0 \%$ serum, M2 MEF, and Coll-1]. FN may also belong to the class 1, although the right-downward shift of vertex (higher $\mathbf{p}$ and lower $\mathbf{q}$ ) (Fig. 6B, C) places this curve slightly below the control curve (Fig. 5D). Of note, all the elements of the class 3 turned out to be also the elements of class 2 , suggesting that the class 3 can be a subgroup of the class 2 .

We also noticed that there are at least two ways to down-regulate Reck at high cell density: (4) to make Reck expression insensitive to cell density [v-Src] (Fig. 5E), and (5) to make the curve more open by increasing the coefficient p [PIKm] (Fig. 5F; Fig. $6 \mathrm{~B}, \mathrm{C})$.

These findings can be explained, if we interpret the data as follows (Fig. 6E): i) Reck is down-regulated when the cells are sparse, ii) Src is involved in such sparsity-dependent Reck down-regulation, iii) serum makes the cells (and/or the Reck gene) less sensitive to cell density and also decreases $r_{\max }$, iv) PI3K is involved in these effects of serum, v) Coll-1 counteracts with serum, and vi) FN counteracts with Src (Fig. 6E). This model predicts that the constitutively active $\mathrm{v}$-Src suppresses Reck expression at any cell density (see Fig. 5E), while PP2 (a Src inhibitor) suppresses Reck down-regulation at low cell density (see Fig. 5A and Fig. 6D (1)). The model also predicts that serum-deprivation, LY294002 (a PIK inhibitor), and Coll-1 up-regulate Reck at lower cell density (see Figs. 4B, 5B, 5C) while the constitutively active PIK makes the cells (and/or the Reck gene) less sensitive to cell density, i.e., lower Reck expression even at high cell density (Fig. 5F). This model is largely consistent with our findings and may serve as a working hypothesis useful for designing further experiments to delineate the molecular mechanism of Reck gene regulation and to find 
effective Reck expression modulators.

This study has also revealed that the $\boldsymbol{r}$ - $\boldsymbol{d}$ curves for M2- MEF are remarkably similar to those for NIH3T3 on Coll-1-coated dishes (Fig. 6A-C). Since MMP-2 can digest Coll-1 [21] and Reck inhibits MMP-2 [9, 22], the Reck over-expression found in these MMP-2-deficient cells may largely be explained by accumulation of Coll-1 or some other molecules having similar effects on Reck.

Although the molecular basis of density-sensing in this system is presently unclear, involvement of the ECM-integrin signaling seems to be a good candidate. The above model (Fig. 6E) predicts that Src activity is suppressed when cell density goes up. Our finding that PP2 and FN share similar effects on the $\boldsymbol{r}$ - $\boldsymbol{d}$ curve is interesting in this respect; accumulation of FN at higher cell density may contribute to the Src-suppression. Similarity between serum-deprivation and Coll-1, on the other hand, may suggest a role for Coll-1 in the regulation of sensitivity to, rather than the sensing itself of, cell density.

FAK is a major signaling molecule of the integrin-based focal adhesion complexes and an important substrate and binding-partner for $\operatorname{Src}[1,5,23,24]$. It also binds, upon tyrosine-phosphorylation, with the $\mathrm{SH} 2$-domain of $\mathrm{p} 85$, the regulatory subunit of PI3K [25]. We initially suspected that FAK might be a common target which integrates the Src-mediated sparsity-signaling and the PI3K-mediated growth factor-signaling. We found, however, that a specific FAK inhibitor (PF573228) [26] had little effects on Reck expression (M. Hatta, unpublished). This, together with other findings presented in this report, suggest that Src and PI3K may impinge on Reck through separate pathways. Our data (Fig. 3C) also suggest that the actions of both pathways on Reck gene probably involve modulation of histone acetylation. Earlier studies indicated that the Ras/Erk pathway suppressed Reck expression and the Sp1 site down-stream of the transcriptional start site was probably involved in this suppression $[17,27,28]$. It is therefore unexpected that the effects Mek1 (Ras/Erk pathway) inhibitor (PD98059) on Reck expression was marginal and that the PI3K pathway seems to be more prevalent in this system. Elucidation of the cis-elements involved in Reck repression mediated by PIK, Src, and their inhibitors Src may provide more clues to the relationship between these two pathways.

In this study, we focused on density-dependent regulation of Reck expression. The method used in this study to analyze the effects of various treatments may be useful in evaluating various other agents, such as chemicals and oncogenes, on Reck expression as well as on the expression of other density-dependent genes. It remains to be tested whether we can illuminate other aspects of Reck regulation by analyzing the effects of various treatments on Reck expression as a function of serum concentration (see Fig. 
2A). It is now clear that Reck is under the control of microRNA-mediated post-transcriptional regulation [29-31]. Some of our data (e.g., Fig. 1D, E) indicated the difference among cell lines in the ratio of Reck protein and mRNA. Whether post-transcriptional regulation, such as differential expression of microRNAs, plays any roles in the density-dependent or serum-dependent Reck regulation is another interesting issue to be addressed in future studies.

\section{Conclusion}

Reck expression is up-regulated at high cell density and after serum-deprivation at both mRNA and protein levels in mouse embryo fibroblasts. Reck expression at low cell density could be up-regulated by PP2, LY294002, and TSA, while Reck expression at high cell density was suppressed by v-Src and an oncogenic mutant of PI3K. Our kinetic studies suggested that Src is involved in the Reck down-regulation under sparse conditions while PI3K is involved in the Reck down-regulation by serum. Fibronectin and type I collagen may counteract with these two regulatory pathways, respectively.

\section{Acknowledgements}

We are grateful to Shigeyoshi Itohara for providing Mmp2-deficient mice, Junseo Oh for the help in establishing M2- MEF, Masahiro Aoki for the PIK3CA-expression vector, and Steve Anderson for 2-1/NP cell line. We also thank Hitoshi Kitayama for technical instructions, Aiko Nishimoto and Hai-Ou Gu for technical assistance, Aki Miyazaki, for secretarial assistance and all the other members of the laboratory for help, discussion, and encouragements. This work was supported by JSPS Grant-in-Aid for Creative Scientific Research and MEXT Grant-in-Aid on Priority Areas.

\section{References}

[1] D.D. Schlaepfer, S.K. Mitra, Curr. Op. Genet. Dev. 14 (2004) 92.

[2] S. Grant, J. Clin. Inv. 118 (2008) 3003.

[3] A.G. Bader, S. Kang, L. Zhao, P.K. Vogt, Nat. Rev. Cancer 5 (2005) 921.

[4] T.L. Yuan, L.C. Cantley, Oncogene 27 (2008) 5497.

[5] G.W. McLean, N.O. Carragher, E. Avizienyte, J. Evans, V.G. Brunton, M.C. Frame, Nat Rev Cancer 5 (2005) 505.

[6] M. Noda, H. Kitayama, T. Matsuzaki, Y. Sugimoto, H. Okayama, R.H. Bassin, Y. Ikawa, Proc. Natl. Acad. Sci. USA 86 (1989) 162.

[7] M. Noda, J. Oh, R. Takahashi, S. Kondo, H. Kitayama, C. Takahashi, Cancer Metastasis Rev. 22 (2003) 167. 
[8] C. Takahashi, Z. Sheng, T.P. Horan, H. Kitayama, M. Maki, K. Hitomi, Y. Kitaura, S. Takai, R.M. Sasahara, A. Horimoto, Y. Ikawa, B.J. Ratzkin, T. Arakawa, M. Noda, Proc Natl Acad Sci U S A 95 (1998) 13221.

[9] J. Oh, R. Takahashi, S. Kondo, A. Mizoguchi, E. Adachi, R.M. Sasahara, S. Nishimura, Y. Imamura, H. Kitayama, D.B. Alexander, C. Ide, T.P. Horan, T. Arakawa, H. Yoshida, S. Nishikawa, Y. Itoh, M. Seiki, S. Itohara, C. Takahashi, M. Noda, Cell 107 (2001) 789.

[10] M. Noda, C. Takahashi, Cancer Sci. 98 (2007) 1659.

[11] T. Muraguchi, Y. Takegami, T. Ohtsuka, S. Kitajima, E.P. Chandana, A. Omura, T. Miki, R. Takahashi, N. Matsumoto, A. Ludwig, M. Noda, C. Takahashi, Nat. Neurosci. 10 (2007) 838.

[12] M. Echizenya, S. Kondo, R. Takahashi, J. Oh, S. Kawashima, H. Kitayama, C. Takahashi, M. Noda, Oncogene 24 (2005) 5850.

[13] S. Kondo, C. Shukunami, Y. Morioka, N. Matsumoto, R. Takahashi, J. Oh, T. Atsumi, A. Umezawa, A. Kudo, H. Kitayama, Y. Hiraki, M. Noda, J Cell Sci 120 (2007) 849.

[14] S. Kawashima, Y. Imamura, E.P. Chandana, T. Noda, R. Takahashi, E. Adachi, C. Takahashi, M. Noda, J Neurochem 104 (2008) 376.

[15] T. Miki, Y. Takegami, K. Okawa, T. Muraguchi, M. Noda, C. Takahashi, J. Biol. Chem. 282 (2007) 12341.

[16] Y. Morioka, J. Monypenny, T. Matsuzaki, S. Shi, D.B. Alexander, H. Kitayama, M. Noda, Oncogene 28 (2009) 1454.

[17] H.C. Chang, L.T. Liu, W.C. Hung, Cell Signal 16 (2004) 675.

[18] H.C. Chang, C.Y. Cho, W.C. Hung, Cancer Res 66 (2006) 8413.

[19] H.C. Chang, C.Y. Cho, W.C. Hung, Cancer Sci 98 (2007) 169.

[20] C.Y. Cho, J.H. Wang, H.C. Chang, C.K. Chang, W.C. Hung, J Cell Physiol (2007).

[21] M.D. Sternlicht, Z. Werb, Annu Rev Cell Dev Biol 17 (2001) 463.

[22] A. Omura, T. Matsuzaki, K. Mio, T. Ogura, M. Yamamoto, A. Fujita, K. Okawa, H. Kitayama, C. Takahashi, C. Sato, M. Noda, J Biol Chem 284 (2009) 3461.

[23] J.T. Parsons, J. Cell Sci. 116 (2003) 1409.

[24] S.K. Mitra, D.A. Hanson, D.D. Schlaepfer, Nat. Rev. Mol. Cell Biol. 6 (2005) 56.

[25] H.C. Chen, P.A. Appeddu, H. Isoda, J.L. Guan, J Biol Chem 271 (1996) 26329.

[26] J.K. Slack-Davis, K.H. Martin, R.W. Tilghman, M. Iwanicki, E.J. Ung, C. Autry, M.J. Luzzio, B. Cooper, J.C. Kath, W.G. Roberts, J.T. Parsons, J Biol Chem 282 
(2007) 14845.

[27] R.M. Sasahara, C. Takahashi, M. Noda, Biochem Biophys Res Commun 264 (1999) 668.

[28] M.C. Hsu, H.C. Chang, W.C. Hung, J Biol Chem 281 (2006) 4718.

[29] G. Gabriely, T. Wurdinger, S. Kesari, C.C. Esau, J. Burchard, P.S. Linsley, A.M. Krichevsky, Mol Cell Biol 28 (2008) 5369.

[30] S.J. Hu, G. Ren, J.L. Liu, Z.A. Zhao, Y.S. Yu, R.W. Su, X.H. Ma, H. Ni, W. Lei, Z.M. Yang, J Biol Chem 283 (2008) 23473.

[31] Z. Zhang, Z. Li, C. Gao, P. Chen, J. Chen, W. Liu, S. Xiao, H. Lu, Lab Invest 88 (2008) 1358. 


\section{Figure Legends}

Fig. 1. Cell density-dependent regulation of Reck expression. (A) Growth curves of NIH3T3 (black diamond) and MEF (white square). The cells were plated onto a series of $35-\mathrm{mm}$ dishes at $5 \times 10^{4}$ cells per dish on day 0 , and the number of cells in two dishes was counted every day after suspension by trypsin-treatment. Medium of remaining dishes was refreshed every day. (B) Reck expression in NIH3T3 detected by immunoblot assay. The cells were harvested at indicated time point, and the level of Reck protein (upper panel) was assessed by immunoblot assay and densitometry. The data were normalized against $\alpha$-tubulin (second panel), divided by the value of day 2 , and shown in bar graph (bottom panel). (C) Reck expression in MEF detected by immunoblot assay. Experiments similar to those described in panel B were performed using MEF cells. (D) The level of Reck protein in NIH3T3 (black bars), MEF (white bars), or M2 MEF (striped bars) at various cell densities. The cells plated at $0.03,0.1$, 0.3 , or $1\left[\times 10^{6}\right.$ cells per $60-\mathrm{mm}$ dish] were harvested after incubation for $48 \mathrm{~h}$, and the level of Reck protein was determined by immunoblot assay and densitometry. The data were first normalized against $\alpha$-tubulin and then divided by the normalized value for the NIH3T3 cells at the lowest cell density. (E) The level of Reck mRNA in NIH3T3 (black bars), MEF (white bars), or M2 MEF (striped bars) at various cell densities. The cells plated onto 60-mm dishes at indicated densities were harvested after incubation for $48 \mathrm{~h}$, and the total RNA was extracted for Reck mRNA measurement by qRT-PCR. The data were first normalized against Hprt and then divided by the normalized value for the NIH3T3 cells at the lowest cell density.

Fig. 2. Serum-dependent regulation of Reck expression. (A) The effects of serum concentration on the level of Reck expression in NIH3T3. NIH3T3 cells plated at $1 \times 10^{5}$ cells per $35-\mathrm{mm}$ dish on the previous day were treated for $24 \mathrm{~h}$ in DMEM containing indicated concentration of serum. The level of Reck protein was estimated by immunoblot assay. (B) The effects of serum-deprivation on the level of Reck mRNA. NIH3T3 cells plated at $1.5 \times 10^{5}$ cells per $35-\mathrm{mm}$ dish on the previous day were incubated in medium containing $10 \%$ serum or in serum-free medium for $24 \mathrm{hr}$, and the level of Reck mRNA was determined by qRT-PCR. (C) Time course of Reck up-regulation after serum withdrawal. NIH3T3 cells plated at $1.5 \times 10^{5}$ cells per $60-\mathrm{mm}$ dish in growth medium on the previous day were incubated in serum-free DMEM for indicated period of time, and the level of Reck protein was assessed by immunoblot assay. (D) Time course of Reck down-regulation in serum-starved cells after serum addition. NIH3T3 cells plated at $1 \times 10^{5}$ cells per $60-\mathrm{mm}$ dish on the previous day were 
serum-starved for $24 \mathrm{~h}$ and refed with growth medium. After incubation for indicated period of time, the cells were harvested for immunoblot assay.

Fig. 3. Effects of various inhibitors on Reck expression. (A) Effects of cell cycle arrest by various treatments on Reck expression. NIH3T3 cells plated at $1 \times 10^{5}$ cells per 60-mm dish on the previous day were incubated in either serum-free DMEM (sf) or in growth serum containing $10 \mathrm{mM}$ hydroxyurea $(\mathrm{HU})$ or $100 \mathrm{ng} / \mathrm{ml}$ nocodazole $(\mathrm{Nz})$ for 16 or $40 \mathrm{~h}$. The cells were also arrested by double-thymidine block (Td) using $2 \mathrm{mM}$ thymidine. Reck expression was estimated by immunoblot assay. Since these treatments may affect the level of a "house keeping gene" used as a loading control, we employed two controls, $\alpha$-tubulin (black bars) and Gapdh (white bars), to confirm the results. (B) Effects of 5-azacitidine (AzC) and Trichostatin A (TSA) on Reck expression. NIH3T3 cells plated at $2 \times 10^{4}$ or $1 \times 10^{5}$ cells per $35 \mathrm{~mm}$-dish on the previous day were treated with $2.5 \mathrm{mM} \mathrm{AzC}$ or $100 \mathrm{nM} \mathrm{TSA}$ for $48 \mathrm{~h}$, and the level of Reck protein was estimated by immunoblot assay. Band intensity (bottom) is the densitometric value first normalized against $\alpha$-tubulin and then divided by normalized value for the control sample [i.e., low density, mock-treated cells] (lane 1). (C) Effects of TSA and serum on Reck expression. NIH3T3 cells plated at $1 \times 10^{5}$ or $5 \times 10^{5}$ cells per $35-\mathrm{mm}$ dish on the previous day were treated with $100 \mathrm{nM}$ TSA for $24 \mathrm{~h}$ in the presence or absence of serum, and the level of Reck protein was estimated by immunoblot assay. Band intensity (bottom) is the densitometric value first normalized against $\alpha$-tubulin and then divided by the normalized value for the cells plated at the lower cell density and incubated in growth medium without TSA. (D) Effects of several inhibitors targeting signaling molecules on Reck mRNA expression. NIH3T3 cells plated at $5 \times 10^{4}$ cells per $60-\mathrm{mm}$ dish on the previous day were treated with GF109203x (GF; 1.5, $4.5 \mu \mathrm{M}$ ), PP2 (1.5, 4.5, $9 \mu \mathrm{M}$ ), PD98059 (PD; 1, 5, $10 \mu \mathrm{M})$, LY294002 (LY; 1, 5, 10 $\mu \mathrm{M})$, Y27632 (Y: 1, 5, 10 $\mu \mathrm{M})$, or Toxin B (TxB; 4, $8 \mathrm{ng} / \mathrm{ml}$ ) for $48 \mathrm{~h}$. The level of Reck mRNA was determined by qRT-PCR, and the data were first normalized against Hprt and then divided by the normalized value for the untreated cells. (E) Co-operative effects of PP2 and LY294002 on Reck mRNA expression. NIH3T3 cells plated at $2 \times 10^{4}$ cells per 35-mm dish were treated with $10 \mu \mathrm{M}$ PP2, $10 \mu \mathrm{M}$ LY294002 (LY), or both for $48 \mathrm{~h}$, and the level of Reck mRNA was determined by qRT-PCR. .

Fig. 4. Density-dependent expression of Reck protein after serum-deprivation and in MEFs. (A) Effects of serum-deprivation on density-dependent Reck expression. NIH3T3 cells plated onto 35-mm dish at indicated cell densities with growth medium 
on the previous day were incubated for additional $24 \mathrm{~h}$ in the presence $(10 \%)$ or absence $(0 \%)$ of serum. Reck and $\alpha$-tubulin were detected by immunoblot assay. (B) The Reck protein versus cell density (or $\boldsymbol{r}_{\boldsymbol{p}}-\boldsymbol{d}$ ) curves for NIH3T3 in the presence (black circles) or absence (white squares) of $10 \%$ serum. The Reck band intensity normalized against $\alpha$-tubulin and then divided by the value of the control sample $\left[5 \times 10^{5}\right.$ cells per dish, $10 \%$ serum] (y-axis) are plotted against cell density (x-axis). Curve fitting revealed that the best fit for both data sets were 2 nd order polynomial equations. The averaged control curve ( $n=6$; see D) is shown in grey dotted line. (C) A quadratic function characterizing the relationship between relative Reck level $(\boldsymbol{r})$ and plating density $(\boldsymbol{d})$. Two forms of quadratic functions (top) and the property of 5 coefficients (a, $, \mathbf{p}, \mathbf{p}, \mathbf{q})$ are described. (D) Variation in 5 coefficients among five control experiments. The $\boldsymbol{r}$ - $\boldsymbol{d}$ approximation curves were obtained from a series control experiments (NIH3T3 in 10\% FCS) and mean \pm s.e.m. of each coefficient is presented. Black bars represent coefficients for Reck protein $(\mathrm{n}=5)$ and white bars for Reck mRNA (n=3). (E) The $\boldsymbol{r}_{\boldsymbol{p}}$ - $\boldsymbol{d}$ curves for NIH3T3 (black circles), MEF (white squares), and M2 MEF (gray triangles). The data shown in Fig. 1D were used to draw approximation curves as described in B.

Figure. 5. Effects of various treatments on the $\boldsymbol{r}-\boldsymbol{d}$ curve. (A) Effects of PP2 on the density-dependent up-regulation of Reck protein in NIH3T3 cells. The $\boldsymbol{r}_{\boldsymbol{p}}$ - $\boldsymbol{d}$ curves for NIH3T3 cells incubated for $48 \mathrm{~h}$ in the absence (black circles) or presence of $10 \mu \mathrm{M}$ PP2 (white squares) are shown. Reference sample: NIH3T3 plated at $5 \times 10^{5}$ per 35-mm dish and incubated in the absence of inhibitor. (B) Effects of LY294002 on the density-dependent up-regulation of Reck protein in NIH3T3 cells. The $\boldsymbol{r}_{\boldsymbol{p}}$ - $\boldsymbol{d}$ curves for NIH3T3 cells incubated for $48 \mathrm{~h}$ in the absence (black circles) or presence of $10 \mu \mathrm{M}$

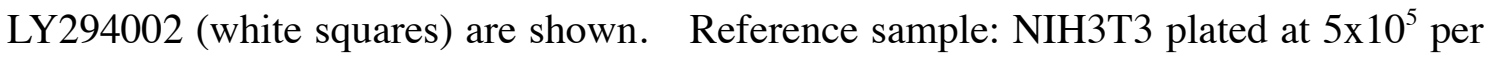
35-mm dish and incubated in the absence of inhibitor. (C) Effects of type I collagen on the density-dependent up-regulation of Reck mRNA in NIH3T3 cells. The Reck mRNA versus cell density (or $\boldsymbol{r}_{\boldsymbol{r}}-\boldsymbol{d}$ ) curves for NIH3T3 cells incubated for $48 \mathrm{~h}$ on regular tissue culture dishes (black circles) or dishes coated with type I collagen (white squares) are shown. Reference sample: NIH3T3 plated at $5 \times 10^{5}$ per $35-\mathrm{mm}$ regular dish. (D) Effects of fibronectin on the density-dependent up-regulation of Reck mRNA in NIH3T3 cells. The $\boldsymbol{r}_{\boldsymbol{r}} \boldsymbol{d}$ curves for NIH3T3 cells incubated for $48 \mathrm{~h}$ on regular tissue culture dishes (black circles) or dishes coated with plasma fibronectin (white squares) are shown. Reference sample: NIH3T3 plated at $5 \times 10^{5}$ per $35-\mathrm{mm}$ regular dish. (E) Effects of v-Src on the density-dependent up-regulation of Reck mRNA in NIH3T3 cells. The $\boldsymbol{r}_{\boldsymbol{r}} \boldsymbol{-} \boldsymbol{d}$ curves for NIH3T3 (black circles) or v-Src NIH (white squares) incubated for 
$48 \mathrm{~h}$ in growth medium are shown. Reference sample: NIH3T3 plated at $5 \times 10^{5}$ per 35-mm dish. (F) Effects of an oncogenic PIKCA mutant (E545K) on the density-dependent up-regulation of Reck mRNA in NIH3T3 cells. The $\boldsymbol{r}_{\boldsymbol{r}}$ - $\boldsymbol{d}$ curves for NIH3T3 (black circles) or PIKm NIH (white squares) incubated for $48 \mathrm{~h}$ in growth medium are shown. Reference sample: NIH3T3 plated at $5 \times 10^{5}$ per $35-\mathrm{mm}$ dish.

Fig. 6. Evaluation of approximation curves. (A) Properties of the $\boldsymbol{r}_{\boldsymbol{p}} \boldsymbol{\boldsymbol { d }} \boldsymbol{\boldsymbol { d }}$ curves. Four coefficients (-a, c, p, q) of the approximation curves shown in Figs. 4B, E and Fig. 5A, $B$ were divided by the value of the control experiments (NIH3T3 in 10\% serum, $n=5$ ). (B) Comparison between the coefficients of $\boldsymbol{r}_{\boldsymbol{r}} \boldsymbol{d}$ curves. Four coefficients of the approximation curves shown in Figs. 5C-F were divided by the value of the control experiments (NIH3T3 in 10\% serum, $n=3$ ). (C) Summary of the trends found in panels A and B. H, high; HH, very high; L, low; LL, very low. Parentheses indicate relatively small changes. (D) Three modes of Reck up-regulation in a lower cell-density range found in this study. (E) A model consistent with our findings. The level of Reck is determined by the quadratic function of cell density. Density dependent up-regulation of Reck (in the left half of the curve) is a consequence of the sparsity-dependent activation (or density-dependent inactivation) of Src. Serum, on the other hand, decreases the sensitivity of Reck expression to cell density, largely through activation of PI3K. Type I collagen may counteract with serum, while fibronectin may regulate the sparsity-dependent effects. 
A

Incubation time [d]

Hatta Fig. 1

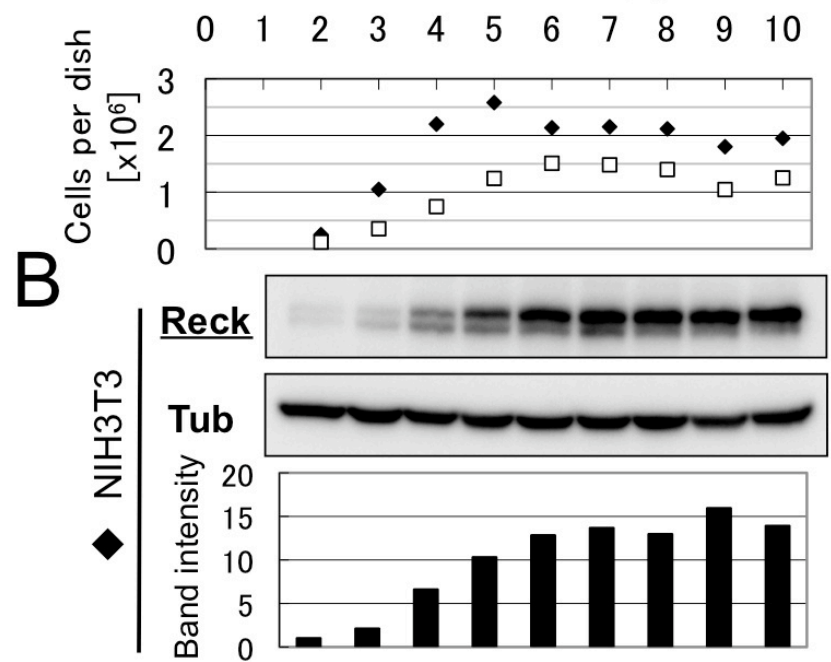

C

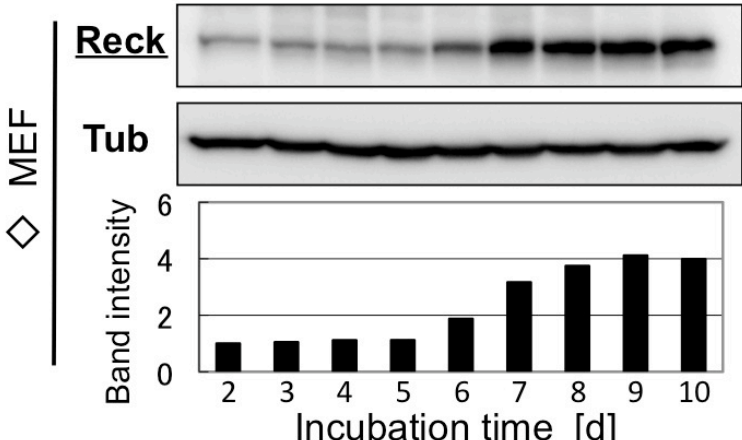

D

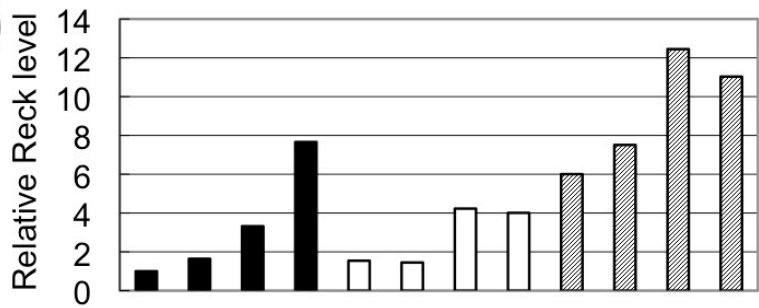

[ell density: $\begin{array}{llllllllllll}0.03 & 0.1 & 0.3 & 1 & 0.03 & 0.1 & 0.3 & 1 & 0.03 & 0.1 & 0.3 & 1\end{array}$
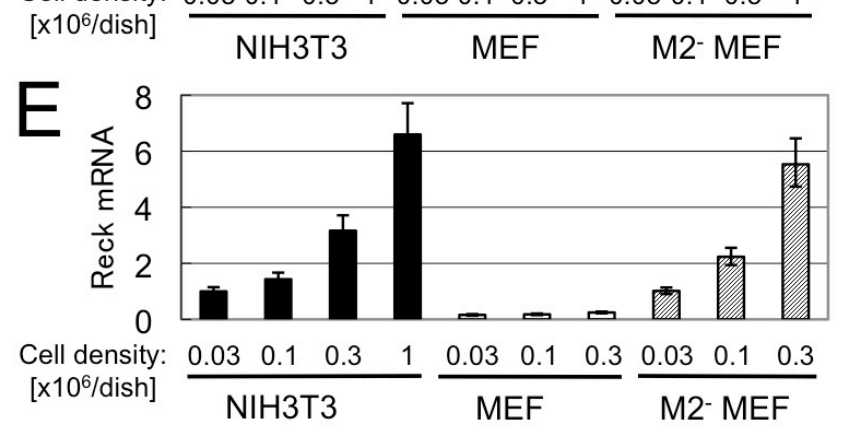


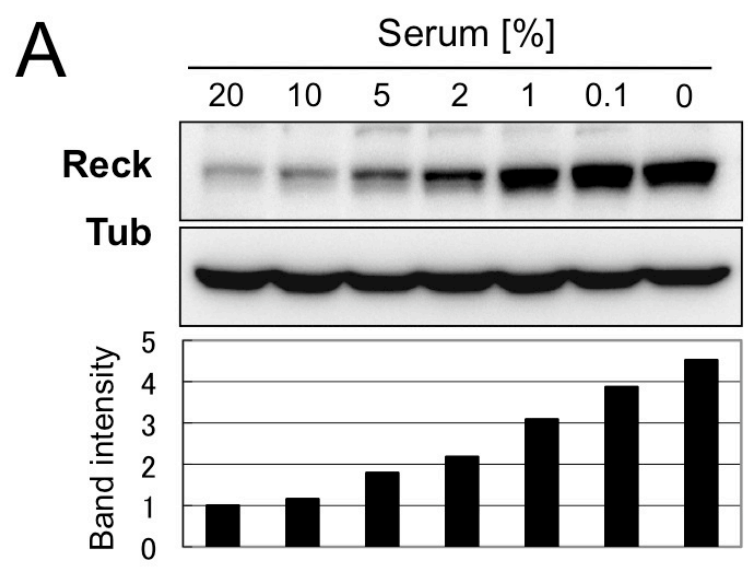

Hatta Fig. 2

B

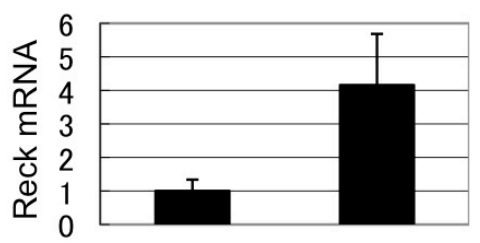

Serum [\%]: 10

0

C

\begin{tabular}{ccccc} 
Time after serum withdrawal $[\mathrm{h}]$ \\
\hline 0 & 3 & 6 & 9 & 24
\end{tabular}

Reck

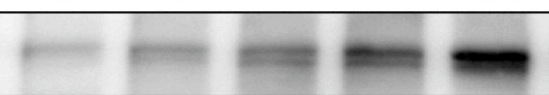

Tub

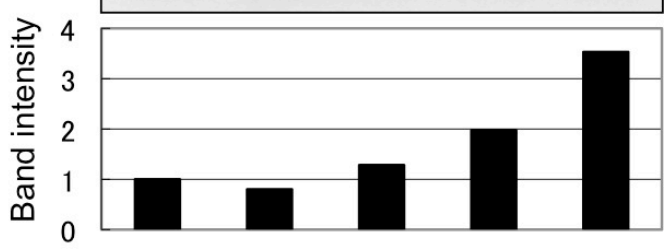

D

\begin{tabular}{llllll} 
Un- & & \multicolumn{4}{c}{ Time after serum addition $[\mathrm{h}]$} \\
\cline { 2 - 6 } starved & 0 & 3 & 6 & 9 & 24
\end{tabular}
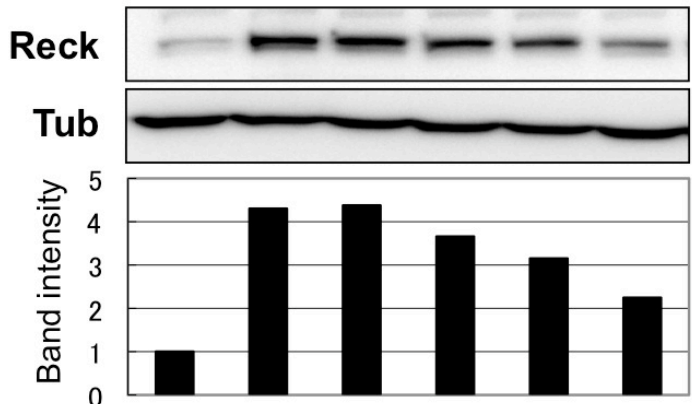


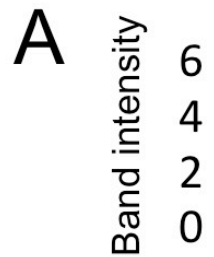

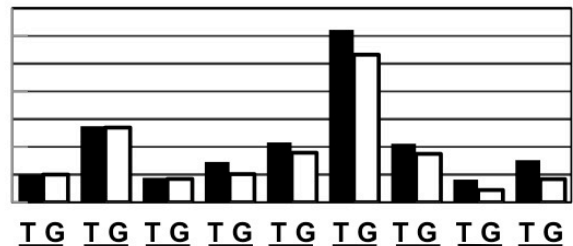

Hatta Fig. 3

Treatment:

TG TG TG TG TG TG TG TG TG

$\frac{-\mathbf{s f} \mathbf{H U ~ N z}}{16 \mathrm{~h}}-\frac{\mathbf{s f} \mathbf{T d ~ H U ~ N z}}{40 \mathrm{~h}}$

B Cell density

Treatment:

0.02

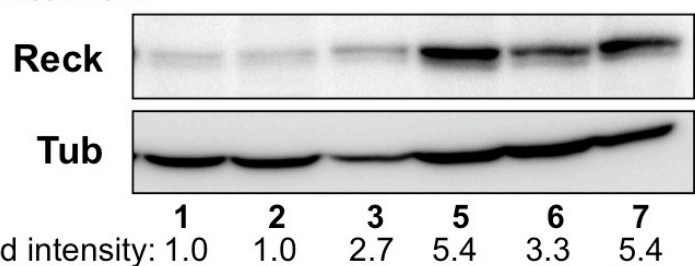

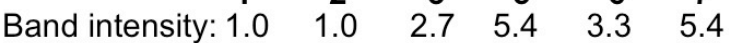

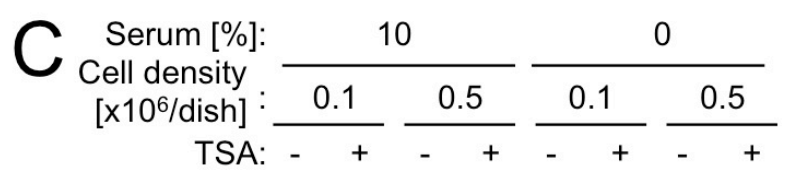

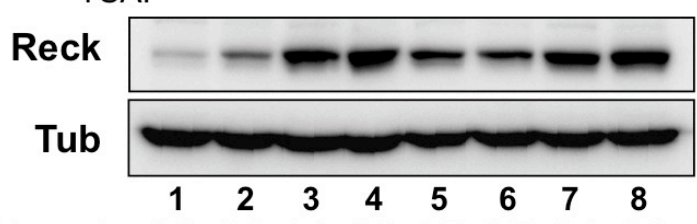

Band intensity: $\begin{array}{llllllll}1.0 & 3.6 & 8.0 & 8.8 & 6.5 & 5.7 & 8.4 & 9.2\end{array}$

D

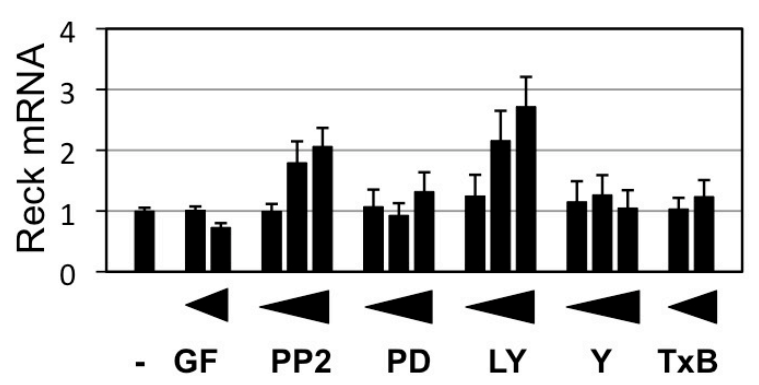

E

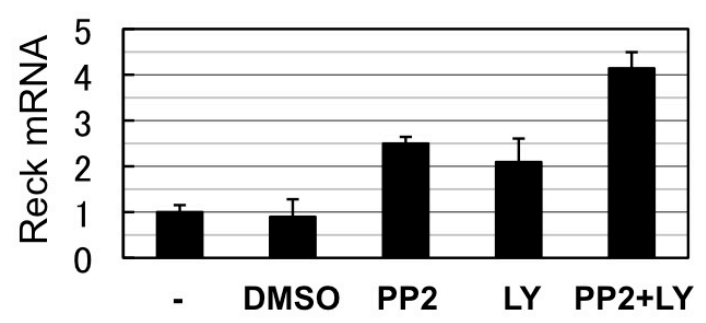


A Serum [\%] :

10

Cell density

[x106/dish] :

0.05

$\begin{array}{lllll}0.1 & 0.25 & 0.5 & 0.7 & 1.0\end{array}$

$0.1 \quad 0.25$

0

\section{Reck}

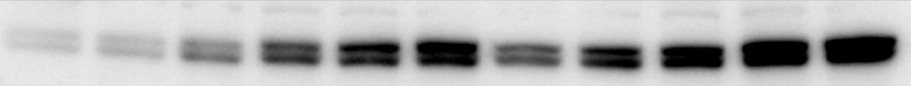

Tub

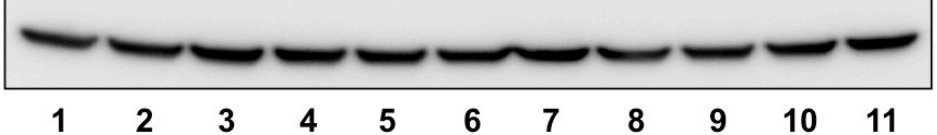

B

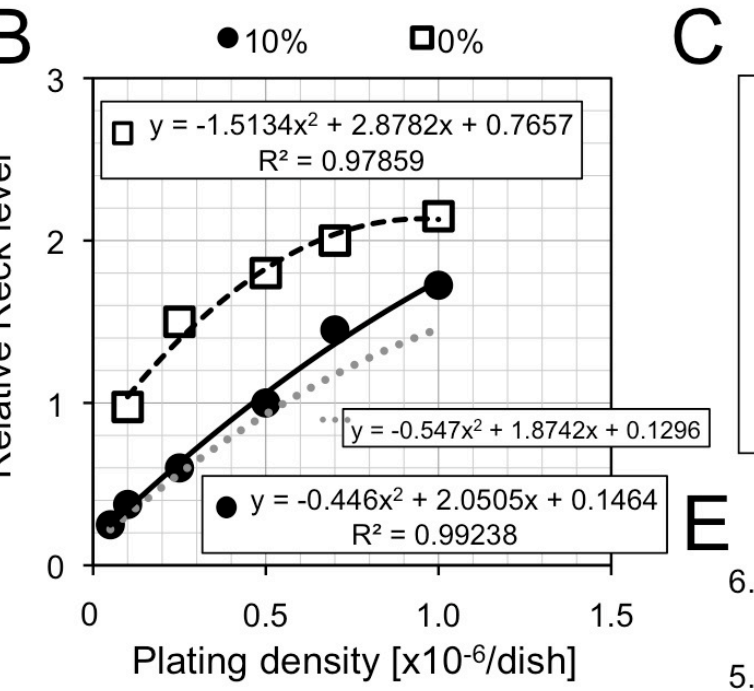

General form: $r=a d^{2}+b d+c$ Standard form: $r=a(d-p)^{2}+q$

$r$ : relative Reck level

$\boldsymbol{d}$ : plating density

a : steepness of parabola

c : $r$ at $d=0$

$\mathbf{p}: \boldsymbol{d}$ yielding $\boldsymbol{r}_{\text {max }}$

q : maximum Reck level $\left(\boldsymbol{r}_{\max }\right)$
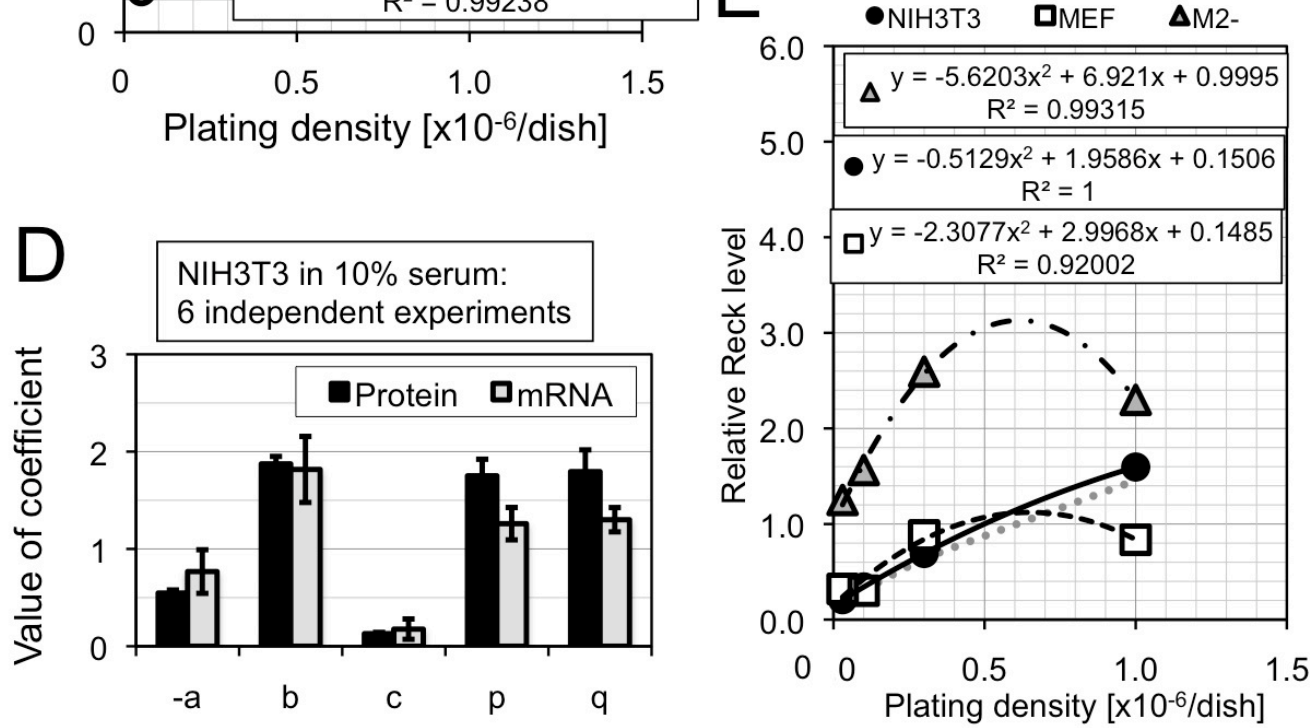


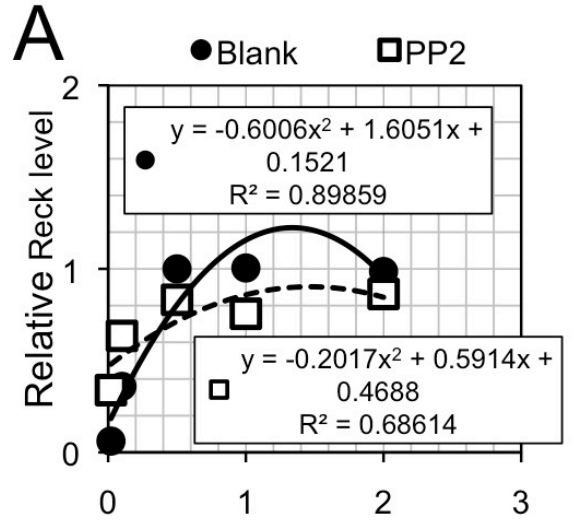

Plating density $\left[\times 10^{-6} / \mathrm{dish}\right]$
B

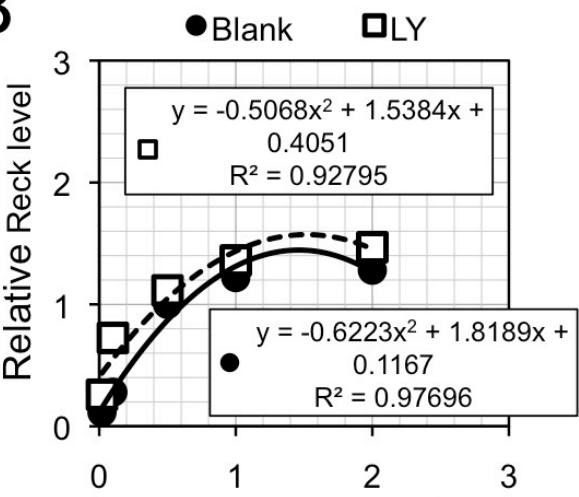

Plating density $\left[\times 10^{-6} / \mathrm{dish}\right]$

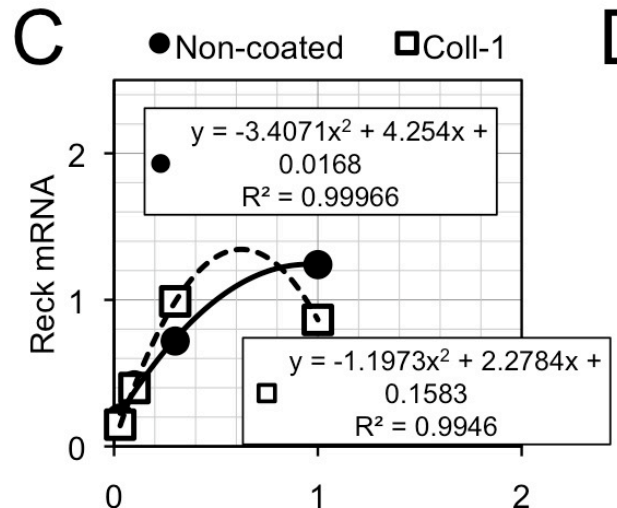

Plating density [x10-6/dish]

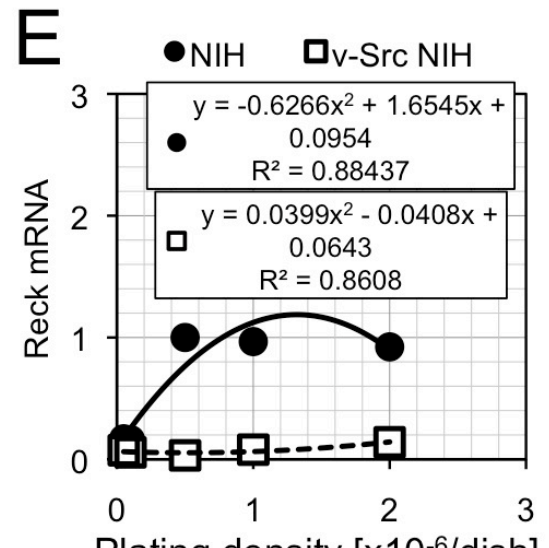

Plating density $\left[\times 10^{-6} /\right.$ dish $]$

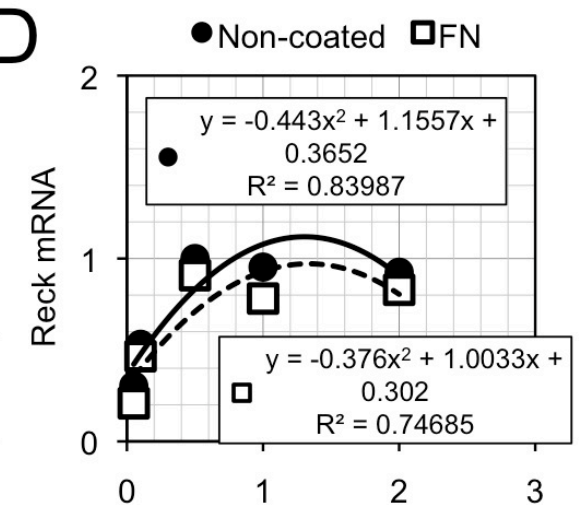

Plating density $\left[\times 10^{-6} / \mathrm{dish}\right]$

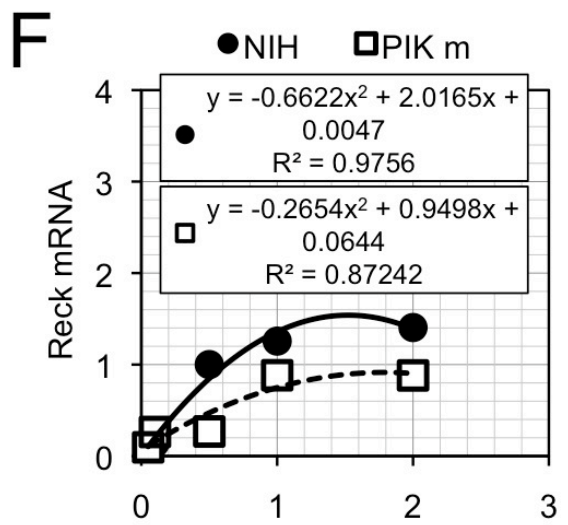

Plating density $\left[\times 10^{-6} /\right.$ dish $]$ 


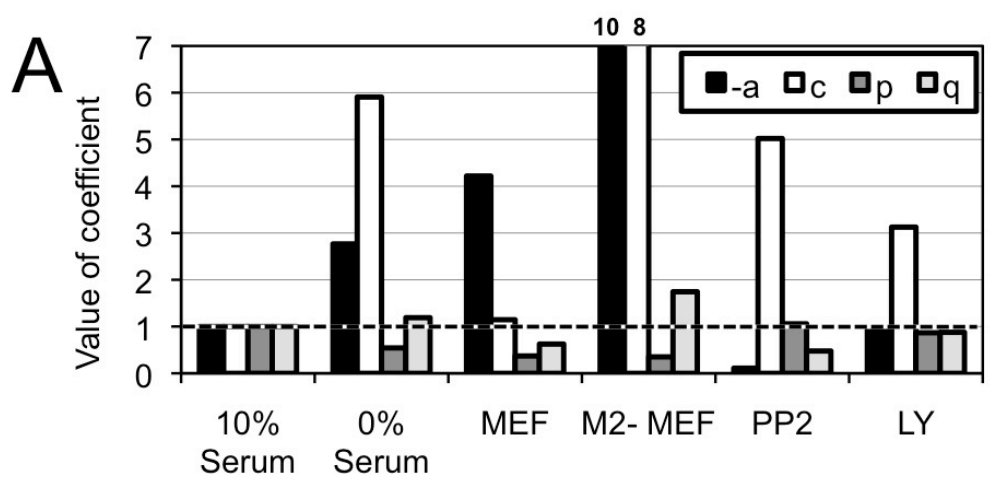

Hatta Fig. 6

B

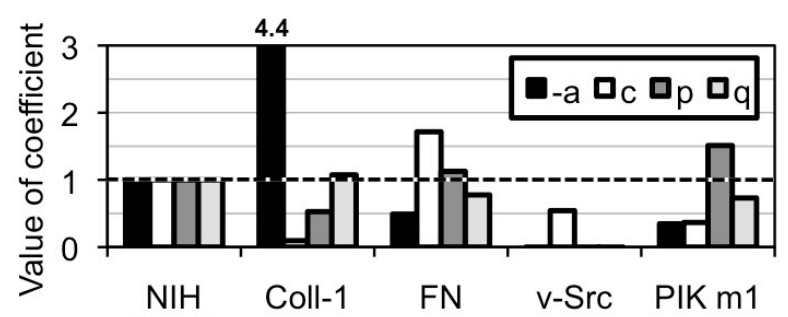

Blank

\begin{tabular}{l|l|l|c|c|c|}
\hline \multicolumn{2}{|c|}{ Coefficient: } & al & c & p & q \\
\hline 1 & $0 \%$ Serum & H & H & L & (H) \\
\hline 2 & MEF & H & & L & L \\
\hline 3 & M2 MEF & HH & H & L & H \\
\hline 4 & PP2 & L & H & & L \\
\hline 5 & LY294002 & & H & (L) & (L) \\
\hline 6 & Coll-1 & H & VL & L & (H) \\
\hline 7 & FN & L & H & (H) & (L) \\
\hline 8 & V-Src & LL & L & LL & LL \\
\hline 9 & PIKm & L & L & H & (L) \\
\hline
\end{tabular}

D

(1) Lower $|a|$

(2) Higher $|a|$, lower $p$

(3) Higher q
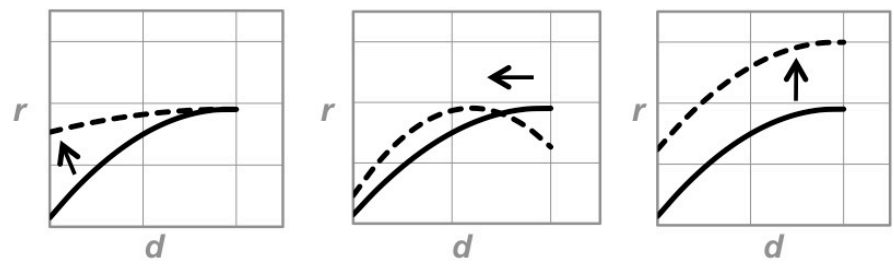

E

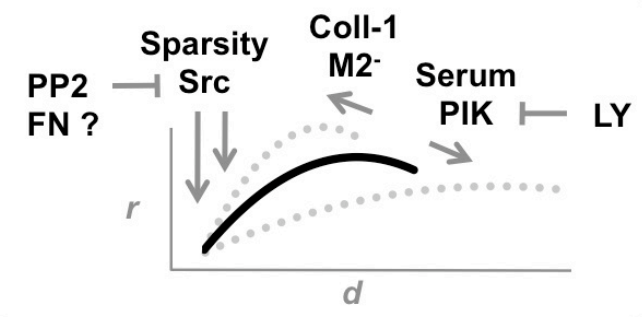

